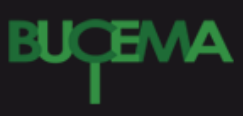

Bulletin du centre d'études médiévales d'Auxerre | BUCEMA

Hors-série $n^{\circ} 7 \mid 2013$

Les nouveaux horizons de l'ecclésiologie : du discours clérical à la science du social

\title{
Quelques notes sur Yves Congar et l'ecclésiologie orientale
}

Pablo Ubierna

(2) OpenEdition

Journals

Édition électronique

URL : https://journals.openedition.org/cem/12866

DOI : $10.4000 /$ cem. 12866

ISSN : 1954-3093

Éditeur

Centre d'études médiévales Saint-Germain d'Auxerre

Référence électronique

Pablo Ubierna, «Quelques notes sur Yves Congar et l'ecclésiologie orientale », Bulletin du centre d'études médiévales d'Auxerre / BUCEMA [En ligne], Hors-série n 7 | 2013, mis en ligne le 29 mars 2013, consulté le 03 mars 2023. URL : http://journals.openedition.org/cem/12866 ; DOI : https://doi.org/ 10.4000/cem. 12866

Ce document a été généré automatiquement le 3 mars 2023.

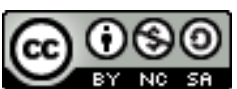

Creative Commons - Attribution - Pas d'Utilisation Commerciale - Partage dans les Mêmes Conditions 4.0 International - CC BY-NC-SA 4.0

https://creativecommons.org/licenses/by-nc-sa/4.0/ 


\title{
Quelques notes sur Yves Congar et l'ecclésiologie orientale
}

\author{
Pablo Ubierna
}

1 Il faut tout d'abord annoncer que le titre est en rapport avec l'approche du père Yves Congar à quelques problèmes de l'ecclésiologie byzantine, notamment la réception des textes historiques sur Photius dans Neuf cents ans après et L'ecclésiologie du Haut Moyen Âge ${ }^{1}$. Yves Congar ne s'est pas occupé de la pensée sur l'Église telle qu'elle s'est développée chez les coptes, les syriaques, les arméniens ou les autres communautés de l'Orient Chrétien '.

2 Les rapports entre la pensée historique et la théologie ont toujours été difficiles au niveau des milieux académiques. L'histoire, écartée des programmes d'études théologiques et de la formation des prêtres, a été une invitée nécessaire et à la fois méconnue dans le monde des théologiens catholiques ${ }^{3}$.

3 Yves Congar a suivi un chemin entièrement différent dont les traces sont connues.

4 Il y a, cependant, deux problèmes à prendre en compte : d'abord, les rapports du père Congar avec l'histoire en tant que théologien et la réception dans l'œuvre d'Yves Congar de l'historiographie sur le monde Chrétien-oriental.

5 Bien que théologien Yves Congar a toujours voulu que son travail soit aussi le métier, d'un historien, d'un médiéviste dont les mérites ont été largement reconnus ${ }^{4}$ au-delà de quelques critiques de la part de spécialistes ${ }^{5}$.

6 L'effort de Congar et de tous les théologiens de sa génération (ou au moins les théologiens issus du Saulchoir et de la Faculté de Théologie de Lyon-Fourvière) était de faire sortir la théologie du cadre traditionnel et, finalement, restreint à des vérités sur un fond d'explication apologétique et de la faire entrer dans l'histoire, y compris l'histoire des institutions et des sociétés. Certainement, l'œuvre de Congar reste pour l'essentiel une histoire des idées mais au moins elle essaie d'établir des liens entre ces idées et les grandes époques historiques pendant lesquelles elles ont été produites ${ }^{6}$.

7 C'est dans l'ancrage des Pères de l'Église que le jeune Congar trouvera les rapports pour une rencontre avec la tradition orientale, encore une fois, de la main de Möhler ${ }^{7}$. Un 
article de 1933 dans Irénikon sur le sujet " pouvoir d'ordre et pouvoir de juridiction dans l'Église" se trouve, sans doute, à l'origine de la pensée congarienne sur une ecclésiologie fondée sur des bases œcuméniques ${ }^{8}$. Il s'agit, à mon avis, d'un texte fondamental parce que le $\mathrm{P}$. Congar s'attaque à des problèmes, comme l'évolution des idées juridiques en Orient et en Occident, qui seront pour lui l'une des raisons les plus importantes pour comprendre l'éloignement des Églises. Alors, il faudrait revenir à ce texte parce que nous y trouvons déjà les arguments qui seront plus tard attaqués après la publication de L'ecclésiologie du Haut Moyen Âge ${ }^{9}$. Or, outre que le manque ou non d'une connaissance juridique de la part de Congar (c'est la base de la critique de Ulmann, dont les opinions ont été, elles aussi, très contestées) je voudrais m'attarder un instant sur les sources et la bibliographie utilisées par Congar pour l'étude de Photius dans son œuvre la plus importante, L'ecclésiologie du Haut Moyen Âge (1968) ${ }^{10}$. D'abord, bien qu'il utilise les classiques du XIX ${ }^{\mathrm{e}}$ siècle comme le Photius de Hergenröther (1867) ou du début du xxe siècle, comme l'article de Jugie publié dans Bessarione, 1919, ou même les deux volumes du Photius de G. Hoffmann (Rome, 1932), l'argumentation de Congar semble reposer sur l'œuvre du P. Fr. Dvornik, The Photian Schism. History and Legend, Cambridge, 1948 que Congar avait fait traduire en français et préfacé en 1950 pour sa collection Unam Sanctam (vol.19). Cependant, cette réception est aussi manquée.

8 Le problème, tel qu'il est résumé par Congar, couvre l'existence d'un débat beaucoup plus large. D'abord, le débat entre le P. Francis Dvornik et le P. Venance Grumel, A. A, notamment sur la place de Photius dans la discussion du filioque, sur la primauté romaine et sur l'annulation (ou non) romaine du concile du $869^{11}$. L'absence des ouvrages du P. Grumel dans la discussion sur Photius est surprenante de plus que le P. Grumel et les Assomptionnistes dirigent à l'époque d'importantes collections et sont à la tête des études byzantines en France ${ }^{12}$. L'autre débat qui n'est pas présent non plus dans ces pages est le débat entre Dvornik et Dölger sur plus ou moins les mêmes problèmes ${ }^{13}$.

9 Néanmoins il faut noter que Grumel et Dölger sont des auteurs mentionnés à plusieurs reprises dans Neuf cents ans après ${ }^{14}$, ouvrage publié quelques années auparavant. Dans ce texte, rédigé en hommage à Dom Beauduin, fondateur d'Amey-Chevetogne en 1953 et publié en $1954{ }^{15}$, l'argument de Congar sur Photius repose sur les livres de Jugie et d'Every ${ }^{16}$. Le livre de Dvornik de 1948, que Congar avait fait traduire et publier dans la collection Unam Sanctam en 1950 est seulement cité, même s'il discute des textes de Dvornik qui sont postérieurs, mais sur des problèmes autres que le schisme photien ${ }^{17}$.

10 La pensée de Congar sur le schisme de Photius s'est consolidée dans les années de guerre et jusqu'à la fin des années 1940. On a vu que la réception de l'œuvre de Dvornik ne fut que très partielle dans son texte de 1954. Quelques aspects seront incorporés dans l'article de la revue Istina de 1959 et c'est cela, dans l'essentiel, qui apparaît dans l'Ecclésiologie en $19688^{18}$. Les auteurs qui sont commentés en 1954 à propos de la pensée politique byzantine (Grumel, Dölger) ne sont point cités à propos du Photius (souvenons-nous que le texte de Congar suit ici de très près les livres de Jugie et d'Every mais il ne mentionne pas celui de Dvornik) et moins encore dans les textes postérieurs à propos de Photius dont l'argument repose, comme nous l'avons dit, sur la, tardive et partielle, réception du livre de Dvornik ${ }^{19}$-très critiqué à la fois par Grumel et Dölgerdans l'œuvre de Congar. 
11 Yves Congar resta toujours un théologien et un historien médiéviste. Dans le cas de l'Église médiévale ses apports sont sans doute de premier ordre et ont continué à vitaliser les études sur l'Église au Moyen Âge ${ }^{20}$. Ses pages sur l'ecclésiologie orientale sont, nous semble-t-il, d'un tout autre ordre. Elles sont plutôt le réflexe de son amour et de son intérêt pour la tradition orthodoxe (même du besoin de compter sur la tradition orthodoxe pour comprendre l'Église) que le produit d'un travail d'historien. De ce point de vue, elles étaient toujours une aide précieuse pour le travail œcuménique et cela justement parce que Congar ne fut pas un historien spécialiste du monde byzantin, mais un théologien dogmatique ${ }^{21}$. Ce fut dans l'œuvre du Père Congar que le monde orthodoxe et la tradition byzantine trouvèrent leur place dans les nouveaux horizons de l'œcuménisme catholique.

\section{NOTES}

1. Il s'agit de présenter ici, à mode d'exercice quelques aperçus sur un problème spécifique. Le travail pourrait s'élargir à l'étude de la réception de la tradition académique sur les Pères, les notions du pouvoir chez les intellectuels de l'époque constantinienne ou les canonistes de l'époque mésobyzantine.

2. Je tiens à remercier ici Dominique Iogna-Prat qui m'a laissé la possibilité de présenter ces pages, produit d'une discussion autour du problème des rapports entre théologie et histoire, tenue lors d'un petit colloque à Buenos Aires sur l'ecclésiologie médiévale en Hommage à Yves Congar en octobre 2010. Étant moi-même historien byzantiniste, et pas du tout un spécialiste de l'œuvre du Père Congar, j'offre ces pages à la discussion en toute humilité puisque sans doute, l'étude des rapports entre l'approche congarienne à l'ecclésiologie médiévale et l'historiographie byzantine a besoin de quelqu'un avec d'autres compétences. La conférence de G. C. CONTICELLO, «L'Orient byzantin dans l'œuvre de Marie-Dominique Chenu ( $†$ 1990) et Yves Congar ( $†$ 1995) » prononcée à l'EPHE-Section des sciences religieuses (Sorbonne) le 14 décembre 2002 ne fut, malheureusement, jamais publiée.

3. En fait les rapports entre histoire et philosophie politique et entre histoire et droit canon ont été aussi très difficiles. Il ne faut que voir la réception, manquée, des études historiques sur le gnosticisme et sur l'empire de Constantin par les philosophes du politique et de toute la discussion sur le "césaropapisme " byzantin par les historiens du droit canon. Dans le monde catholique, l'essor, au moins dans certains cercles, des études historiques pendant les $\mathrm{XVI}^{\mathrm{e}}$ et $\mathrm{XVII}^{\mathrm{e}}$ siècles, et même dans le XVIII ${ }^{\mathrm{e}}$ siècle, tomba dans l'aliénation des $\mathrm{XIX}^{\mathrm{e}}$ et $\mathrm{XX}^{\mathrm{e}}$ siècles, ce qui a toujours posé un problème pour une foi qui se veut enracinée dans l'histoire. La tradition gallicane, l'érudition des Messieurs de Port Royal sont bien connues, surtout en Sorbonne, dont un Centre porte le nom de Lenain de Tillemont. Cf. le recueil d'articles de B. NEVEAU, Érudition et Religion aux XVII et XVIII siècles, Paris, 1994. Je me permets de citer aussi un ouvrage, tout récent, d'un chercheur français, sur les traditions d'outre-Manche. Cf. J.-L. QUANTIN, The Church of England and Christian Antiquity. The construction of a Confessional Identity in the 17th century, Oxford, 2009. Toute la discussion autour du Modernisme peut être comprise comme une discussion sur la place de l'histoire dans la théologie catholique. Cf. Fr. LAPLANCHE, La crise de l'origine. La science catholique des Évangiles et l'histoire au XXe siècle, Paris, 2006. 
4. La place de L'ecclésiologie du Haut Moyen Âge (1968) et de L'Église de saint Augustin à l'époque moderne (1970) dans le cadre de l'historiographie sur l'Église médiévale a été récemment retracée par J. W. o'MALLEY, "Yves Congar as Historian of Ecclesiology », in G. FLYNN (éd), Yves Congar. Theologian of the Church, Louvain, 2005, p. 229-249 (Louvain Theological and Pastoral Monographs).

5. Cf. G. ARNALDI, "Congar et l'ecclésiologie du Haut Moyen Âge. Quelques réflexions 1», in A. vaucheZ (dir.), Cardinal Yves Congar 1904-1995, Paris, 1999, p. 27-39 et O. CAPITANI, «Congar et l'ecclésiologie du Haut Moyen Âge. Quelques réflexions 2», in Ibid., p. 41-49. Voir aussi le compte rendu de W. ULmanN à L'Ecclésiologie, Journal of Theological Studies 21 (1970).

6. Cf. J.W. O'MALleY, op.cit., p. 233. Le problème épistémologique que pose la catégorie historiographique d'« histoire de l'Église » n'a pas encore été étudié profondément. En tout cas, nous pouvons avancer qu'il ne s'agit pas seulement d'un problème de la science catholique mais qu'il se trouve aussi dans les ouvrages issus de la tradition protestante. Cf. R. WiLLiams, "Newman's Arians and the Problem of Method in Doctrinal History », in I. KER, A. G. HILL (éds.), Newman after a Hundred Years, Oxford, 1990, p. 263-285. La réception de Newman par Congar, notamment dans La Foi et la Théologie (1950), Jalons pour une théologie du laïcat (1953), La Tradition et la Vie de l'Église et dans la rédaction des premiers brouillons de la Constitution Dei Verbum, a été largement étudiée dans les aspects plus dogmatiques, il manque encore une étude approfondie des rapports entre les deux théologiens au niveau de l'importance de la connaissance historique pour les théologiens, surtout si nous tenons compte de l'influence de Möhler, à la fois comme ecclésiologue et comme patrologue, sur Congar c'est évident depuis ces années d'études avec Chenu. Cf. en dernier lieu, A. MESZAROS, "'Haec Traditio proficit': Congar Reception of Newman in Dei Verbum, Section 8 ", in The New Blackfriars, 92 (2011), p. 247-248. Les rapports entre la tradition anglicane et l'orthodoxie, ainsi que les sources historiques et patristiques de leurs revendications étaient toujours présents dans le travail de Congar. Cf. Y. CONGAR, « Positions des orthodoxes et des anglicanes ", in Irénikon 23 (1950), p. 302-328. Contrairement à o'MALLEY (p. 233-234) nous pensons qu'il est tout à fait surprenant que l'œuvre d'Yves Congar ne semble pas avoir reçu l'influence de l'historiographie française la plus moderne. Les noms des grands médiévistes français sont presque tous absents de l'œuvre de Congar. S'il est bien vrai que la place de l'histoire est importante dans l'éducation française -et de ce point de vue, avec O'Malley nous pouvons voir l'influence de l'histoire chez les philosophes et théologiens français- il y a toujours un grand écart entre « donner une place à l'histoire » et « avoir des rapports intellectuels avec les historiens de profession ». Ceci est dit d'une façon générale puisque dans quelques passages, Congar fait appel à Marc Bloch, par exemple, pour contextualiser la discussion sur la "Thèse Pirenne » (Cf. Y. CONGAR, Neuf cents ans après, chap. 2, n. 39) ou La Méditerranée de Fernand Braudel à propos de la prise de Constantinople et des $\mathrm{XV}^{\mathrm{e}}-\mathrm{XVI}^{\mathrm{e}}$ siècles. Cependant, nous ne pouvons pas parler de l'existence d'un dialogue avec l'historiographie française.

7. La place de Möhler comme patrologue serait à nouveau présente dans ses articles pour la revue Irénikon du Monastère œcuménique de Chevetogne. Cf. Y. CONGAR, "La pensée de Möhler et l'ecclésiologie orthodoxe ", in Irénikon 12 (1935), p. 321-329 (courte présentation de l'article du P. ROUZERT, «L'Unité organique du catholicisme d'après Mohler») et «La signification œcuménique de l'œuvre de Möhler », in Irénikon 15 (1938), p. 113-130. Sur l'importance de Möhler pour Congar voir aussi Y. CONGAR, «L'Esprit des Pères d'après Möhler », in SVS 55 (1938), p. 1-25. Sur les contacts entre Congar et Chevetogne, voir E. LANNE, " Le Père Congar et la revue Irénikon », in A. VAUCHEZ, op. cit., p. 105-115. Cf. aussi Y. CONGAR, «Conscience ecclésiologique en Orient et en Occident du $\mathrm{VI}^{\mathrm{e}}$ au XI ${ }^{\mathrm{e}}$ siècle », in Istina 6 (1959), p. 187-236.

8. Y. CONGAR, «Ordre et juridiction dans l'Église », in Irénikon 10 (1933), p. 22-31, 97-110, 243-252, 401-408. 
9. Cf. W. ulmanN, op. cit., p. 224-225. Or, il ne faut pas oublier que c'était bien Ulmann l'objet de la critique de Congar à propos de ses opinions sur l'existence d'une idée théocratique chez les papes du Moyen Âge. Cf. Y. CONGAR, L'ecclésiologie, op. cit., p. 239 n. 42.

10. Cf. p. 216 et suiv.

11. Cf. d'abord le compte rendu du P. Grumel à l'œuvre de Dvornik in REB 10 (1952), p. 282-283. Cf. aussi V. GRUMEL, «Le 'filioque' au Concile Photien de 879-880 et le témoignage de Michel d'Anchilaios ", in EO 29 81939), p. 257-264; "Y eut-il un second schisme de Photius?», in R.Sc.Phil.et Théol. 22 (1933), p. 432-457; «Le schisme de Grégoire de Syracuse", in EO 39 (1940-42), p. 257-267; « Photius et l'addition du Filioque au symbole de Nicée-Constantinople », in REB 5 (1947), p. 218-234. Voir aussi P. GEMEINHARDT, Die Filioque-Kontroverse zwischen Ost-und Westkirche im Frühmittelalter, Berlin, 2002, p. 260-264 sur la discussion Dvornik-Grumel. Il s'agit ici de présenter le problème de la réception d'une discussion sur le filioque dans les œuvres plus historiques de Congar. Les théologiens considèrent que toute discussion sur le filioque ne peut passer du dialogue avec l'œuvre de Congar sur le Saint-Esprit. Cf. E. T. Groppe, Yves Congar Theology of the Holy Spirit, Oxford-New York, 2004.

12. L'Institut Français d'Études Byzantines (continuation des Échos d'Orient fondée par les Assomptionnistes à Kadiköy, banlieue d'Istanbul entre 1897 et 1942), la Revue des Études Byzantines et la Revue de l'Orient Chrétien. Ils participaient étroitement aussi dans les projets divers comme la «Bibliothèque Byzantine » publiée sous la direction de Paul Lemerle.

13. Cf. F. DÖLGER, « Rom in der Gedankenwelt der Byzantiner », in Zeits. f. Kirchengesch. 56 (1937), p. 1-42 et dans le recueil F. DÖLGER, Byzanz und die europäische Staatenwelt : Ausgewählte Vorträge und Aufsätze, Ettal, 1953, p. 70-115. La discussion contre Dvornik est centrée sur l'authenticité du petit traité sur la fondation apostolique de Constantinople, attribué à Photius (cf. Byzanz, p. 100-115 et p. 312-315; pour les opinions de Dvornik, voir Photian Schism, p. 80 et suiv.) Dvornik va finalement publier une œuvre importante sur le sujet, F. DVoRNIK, The Idea of Apostolicity in Byzantium and the Legend of the Apostle Andrew, 2 vols, Washington, 1958, texte sans réception dans l'œuvre de Congar.

14. Y. CONGAR, "Neuf cents ans après. Notes sur le 'Schisme oriental' ", in 1054-1954 : L'Église et les Églises. Neuf siècles de douloureuse séparation entre l'Orient et l'Occident. Études et travaux sur l'Unité chrétienne offerts à Dom Lambert Beauduin, vol. I, Chevetogne, 1954 (Collection Irénikon). Nous citons d'après la traduction anglaise After Nine Hundred Years. The background of the Schism between the Eastern and Western Churches, New York, 1959. Le texte prépare l'article de la revue Istina de 1959 sur l'ecclésiologie orthodoxe.

15. Cf. E. LANNE, op. cit., p. 110-111.

16. M. JUGIE, Le Schisme byzantin. Aperçu historique et doctrinal, Paris, 1941 ; G. EVERY, The Byzantine Patriarchate 451-1204, Londres, 1947.

17. Le texte de Dvornik est mentionné à la fin du chapitre II mais seulement la Préface écrite par Congar, un texte à portée théologique sur le besoin de laisser de côté l'éloignement entre les deux traditions ecclésiales. Le livre est à nouveau mentionné, toujours au passage, dans les notes 51,76 et 79 du chapitre IV, toujours dans la version anglaise. Il est très intéressant de noter que dans la note 16 du chapitre IV, sur les textes recommandés pour l'étude du schisme, Congar mentionne outre que le classique de Hergenröther, les textes de Duchesne, Bréhier, Jugie sans doute, mais aussi l'article d'Ivanka dans Irénikon 9 (1932) mais non le livre de Congar. Par contre Congar suit la discussion sur les rapports entre l'Empereur et l'Église à Byzance au plus proche (c'est-à-dire, sur un autre sujet, avec un autre fichier) en commentant un article de Dvornik de 1951 («Pope Gelasius and Emperor Anastasius I», in BZ 44 [1951], p. 111-116) et le mettre en rapport avec un article de W. SETTON de 1947 ("Constantine as a Bishop», in JRS 37 [1947], p. 127-131 et les critiques à l'article de Setton faites par V. LAURENT dans la REB 6 (1948), p. 115-116. À cet endroit-là Congar cite l'article de F. DöLGER, « Rom in der Gedankenwelt... » dans 
le cadre d'une bibliographie plus traditionnelle pour un théologien (Treitinger, Peterson, Arquillière).

18. Surtout dans la Section $C$ "L'Orient accord et divergences ecclésiologiques avec Rome et l'Occident ». Les considérations de Dvornik dans son texte sur Photius furent incorporées dans les paragraphes sur le pape Nicolas et l'Église orientale, p. 216 et suiv. la rédaction du texte fut finie avant 1966 parce que l'auteur n'a pas pu tenir compte du livre de Dvornik Early Christian and Byzantine Political Philosophy. Origins and Background, Washington, 1966. Voir L'Écclésiologie, p. 346, n. 87.

19. La place du livre de Dvornik dans une « réhabilitation » de Photius par un auteur catholique est en soi un problème historiographique très intéressant.

20. Ici l'influence de Congar est directe. Il s'agit de ses propres recherches et comment elles furent incorporées dans sa pensée œcuménique. Un cas tout à fait différent, par exemple, de la réception -et postérieurement, vulgarisation- de l'œuvre de Brian Tierney sur l'Église médiévale et sur le conciliarisme par Hans Küng.

21. Il ne faut se souvenir que des longues années d'amitié avec les moines de Chevetogne, ses articles dans Irénikon et Istina, et aussi de l'intérêt dans le monde anglo-saxon pour Cent ans après. Les rapports entre Congar et la théologie orthodoxe contemporaine (John Zizioulas par exemple) restent toujours dans une approche commune au problème de l'Esprit et de la personne (cf. n. 11 apud). Voir en dernier lieu, J. SKIRA, «Breathing with two Lungs. The Church in Yves Congar and John Zizioulas ", in M. S. ATTRIDGE, J. Z. SKIRA (éds.), In God Hands: essays on the Church and Ecumenism in Honour of Michael H. Fahey SJ, Louvain, 2006. La lecture de la version anglaise de Neuf cent ans après fut aussi un tournant dans la vie du jeune Kallistos Ware. Cf. K. WARE, « Préface », in G. FLYNN (éd.), op. cit., p. 31-32.

\section{INDEX}

Mots-clés : ecclésiologie, œcuménisme, Congar Yves

\section{AUTEUR}

\section{PABLO UBIERNA}

Université de Buenos Aires-CONICET 